Le Swiss Quality Award a pour but de donner des impulsions afin de poursuivre le développement de la qualité dans le domaine de la santé. Pour cela, vous trouverez sur www.swissqualityaward.ch $\rightarrow$ Les projets un large choix de projets en faveur de la qualité des soins soumis lors des éditions précédentes du Swiss Quality Award. Que vous cherchiez de l'inspiration pour développer votre propre projet, ou de nouveaux contacts afin d'échanger avec d'autres spécialistes, n'hésitez pas à consulter le site du concours. Les nombreux projets qui nous sont parvenus ces dernières années montrent bien que la notion de qualité est vécue de manière très différente dans l'activité médicale au quotidien. Vous avez élaboré un projet novateur en faveur de la qualité? Alors inscrivez-vous au Swiss Quality Award d'ici au 8 janvier 2016!

Dr Christoph Bosshard, membre du Comité central de la FMH, responsable du département Données, démographie et qualité / Académie suisse pour la qualité en médecine (ASOM)

\title{
Mise au concours du Swiss Quality Award 2016
}

\section{Michelle Gerber}

lic. phil. hum., collaboratrice scientifique FMH, division Données, démographie et qualité (DDQ) / Académie suisse pour la qualité en médecine (ASQM)

C'est parti: dès à présent, vous pouvez inscrire votre projet en faveur de la qualité au Swiss Quality Award 2016. Aux côtés de la SQMH et de l'IEFM, la FMH/ASQM remettra une nouvelle fois ce prix en 2016 pour récompenser l'engagement des pionnières et des pionniers qui ont œuvré au développement de la qualité dans le domaine de la santé.

$\mathrm{Au}$ cours des différentes éditions du Swiss Quality Award, le nombre élevé de participants (ils étaient 67 lors de la dernière édition) a confirmé l'important engagement des spécialistes de la santé en faveur de la qualité des soins. En 2016, les trois organismes responsables que sont la Société suisse pour le management de qualité dans la santé (SQMH), l'Institut pour la recherche évaluative en médecine (IEFM) de l'Université de Berne et la Fédération des médecins suisses (FMH) entendent une nouvelle fois faire connaître au plus grand nombre les projets novateurs en faveur de la qualité. Pour cela, ils offrent la possibilité non seulement aux projets lauréats mais également à d'autres projets pertinents d'être publiés sur le site Internet du Swiss Quality Award afin de gagner en visibilité, nourrir le débat spécialisé et créer le lien entre les responsables qualité du domaine de la santé, ce qui profitera non seulement aux professionnels et institutions de la santé, mais également aux patients.

Le Swiss Quality Award est décerné dans trois catégories: «hospitalier», «ambulatoire» et «intersectoriel»,

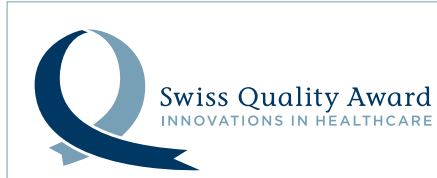

chacune dotée d'un prix de 10000 francs. De plus, un prix supplémentaire d'une valeur de 2000 francs, le «Swiss Quality Poster-Award», reviendra à l'un des meilleurs projets soumis. Qu'ils concernent les cabinets médicaux, les soins à domicile, les cliniques ou les établissements de soins, les projets relatifs à la qualité peuvent apporter de grands bénéfices dans tous les domaines. Tous les projets dédiés à la qualité peuvent être soumis, peu importe la méthode utilisée, l'essentiel est qu'ils contribuent à améliorer la qualité des soins dans le domaine de la santé. Même les petits projets ont toutes leurs chances. S'ils apportent une amélioration durable pour les patients, ils représenteront eux aussi une source d'inspiration pour les autres organisations.

Une idée a-t-elle fait ses preuves dans votre cabinet, votre clinique, votre organisation ou établissement de soins et vous souhaiteriez la partager avec le plus grand nombre? Alors n'hésitez pas à participer au Swiss Quality Award 2016! Rappelons que ce n'est pas la taille du projet qui est déterminante mais son efficacité.

Vous avez la possibilité de vous inscrire en ligne sous: www.swissqualityaward.ch $\rightarrow$ L'inscription. Le délai d'inscription est fixé au 8 janvier 2016.

La remise des prix aura lieu le $1^{\text {er }}$ juin 2016 à Soleure, lors du Symposium national pour la gestion de la qualité dans le secteur de la santé. Vous trouverez de plus amples informations sur le Swiss Quality Award sous www.swissqualityaward.ch 